\title{
Granular dampers in microgravity: sharp transition between modes of operation
}

\author{
Achim Sack $^{1} \cdot$ Kit Windows-Yule $^{2} \cdot$ Michael Heckel $^{1} \cdot$ Dominik Werner $^{2} \cdot$ Thorsten Pöschel $^{1}$
}

Received: 20 November 2019 / Published online: 6 May 2020

(c) The Author(s) 2020

\begin{abstract}
When a container filled with granular material is subjected to sinusoidal vibration in microgravity, dependent on the amplitude of the oscillation, the granulate may exhibit one of two distinct dynamical modes: at low amplitude, a gas-like state is observed, where the particles are relatively homogeneously distributed within the container, almost independent of the phase of the oscillation. In contrast, for large amplitude, collective motion of the particles is favoured, termed collect-andcollide regime. Both regimes are characterized by very different dissipation characteristics. A recent model predicts that the regimes are separated by a sharp transition due to a critical amplitude of the vibration. Here we confirm this prediction of a sharp transition and also the numerical value of the critical amplitude by means of experiments performed under conditions of weightlessness.
\end{abstract}

Keywords Granular matter $\cdot$ Granular damper $\cdot$ Impact damper $\cdot$ Dissipation $\cdot$ Microgravity $\cdot$ Weightlessness $\cdot$ Parabolic flight

\section{Introduction}

\subsection{Granular dampers}

When granular particles collide with one another or with container walls, they dissipate mechanical energy. This fundamental property which, in fact defines the term granular particle originates from the fact that grains are macroscopic or thermodynamic bodies with many internal degrees of freedom. In contrast, molecular particles with only a few internal degrees of freedom cannot dissipate energy but quickly reach thermodynamic equilibrium where on average, the energy is evenly distributed among all degrees of freedom. The dissipative properties of granular matter do

Electronic supplementary material The online version of this article (https://doi.org/10.1007/s10035-020-01017-x) contains supplementary material, which is available to authorized users.

Thorsten Pöschel

thorsten.poeschel@fau.de

1 Institute for Multiscale Simulation, Universität ErlangenNürnberg, Cauerstraße 3, 91058 Erlangen, Germany

2 School of Chemical Engineering, University of Birmingham, Edgbaston, Birmingham B15 2TT, UK not only give rise to a rich physical phenomenology but are also of significant interest for vibration damping in technical applications, ranging from medicine [1, 2], through recreational sports [3] to construction [4-7].

In their most basic form, granular vibration dampers comprise an assembly of particles housed in a sealed container or cavity. When exposed to oscillations of an adequately high vibrational energy ( $\bar{E} \propto \omega^{2} A^{2}$, where $\omega$ and $A$ are, respectively, the angular frequency and amplitude of the vibration), the particles will overcome the sedimenting force of gravity, collide with one another and the walls of the housing container and, consequently, dissipate energy. This dissipation attenuates the vibration and, thus, damps the vibrating system [8-14]. In order to isolate and better understand these phenomena, it is often valuable to study the dynamics of these systems in a zero-gravity environment, where complexities due to gravitational acceleration, and the resultant spatial heterogeneities ${ }^{1}$ and strong particle-wall frictional interactions induced thereby are limited. It should be noted, however, that the simplified, gravity-free systems studied here are not without direct real-world application - see, for

\footnotetext{
${ }^{1}$ Note that while the zero-g environment reduces spatial homogeneities due to gravity, that is not to say that spatial heterogeneities cannot develop in such microgravity conditions-as illustrated, for example, in reference [15].
} 
example, the Russian EVA hammer, used for work outside the MIR space station.

\subsection{Vibrated granular systems in microgravity}

The dynamics of vibrated granular media in microgravity have been the subject of academic study for more than two decades. Early work by Falcon et al. $[15,16]$ showed that a dilute granular medium in microgravity will maintain a relatively homogeneous density distribution, whereas a denser system will typically collapse into a single dense cluster. Low-gravity environments have also proven a valuable means of studying fundamental phenomena and properties of granular media, such as velocity-dependent restitution coefficients [17, 18], wave propagation [19], and the coupling of rotational and translational energy [20-23], an important problem in the field of granular physics, where equipartition cannot be assumed [24, 25]. Indeed, the simplicity of microgravity systems makes them ideal for investigating various non-classical behaviours exhibited by granular gases [26, 27], in particular their well-known non-Gaussian velocity distributions [28, 29]. More recently, significant interest has developed in the study of the phase transitions of vibrated dry granular media in microgravity [30-34], and it is one such transition which forms the focus of the present work.

\section{Modes of operation of granular dampers}

Previous experiments [35-37] revealed two very distinct dynamical modes of granulate in a granular damper when subjected to sinusoidal motion, $A(t)=A \cos \left(\omega t+\varphi_{x}\right)$, in weightlessness: at low driving amplitudes, the granulate is found to exist in a gas-like state, where the particles near-homogeneously fill the available space. In contrast, at large amplitudes the particles move coherently in synchrony with the external vibration which suggests the modeling of the granulate's motion as a single completely inelastic quasi-particle oscillating between the walls of the container. The current mode of operation is independent of the frequency but depends only on the amplitude of the vibration. The threshold amplitude, $A_{0}$, discriminating the modes depends only on the physical free space inside the container, i.e. the space not occupied by particles.

A similar transition between a gas-like and a solid-like state has also been observed under gravity [38]. However, unlike in the microgravity experiments described in [35-37] which suggest a single phase boundary between the two states, these experiments showed strong hysteresis [38]. Other experiments under Earth's gravitational conditions also demonstrated more complex dynamics, such as a liquid-like 'sloshing' motion [39] that is not observed in microgravity experiments.

The previous microgravity experiments discussed above focused on the highly dissipative collective regime, while the region near the threshold was studied insufficiently. In the current paper we address this issue, exploring in detail the damping properties in the gas regime and near the critical amplitude. A few measurements for the collective regime have been repeated for calibration.

In order to understand the key rôle of the threshold separating the two regimes, we first need to understand the main differences between said regimes [35, 36]: at low driving amplitudes, the particles fill the whole container nearly homogeneously with the exception of a gradient which forms where the walls of the container sweep the space. Particles in the zones which are swept by walls once per period get accelerated in the process and successively lose their kinetic energy in the subsequent cascade of dissipative particle-particle and particle-wall collisions. Consequently, at low amplitudes in each period only a small fraction of all particles comes into contact with the wall and, therefore, the gas state is characterized by a relatively low energy dissipation rate.

In contrast, at large driving amplitude, particles are observed to undergo collective motion: during the inward stroke of a wall, the particles are gathered on the inwardaccelerating wall due to a granular collapse [40, 41]. At the point of inflection of the motion, the bulk of particles leaves the wall as an agglomerate with only a small variation in velocity between its constituent particles. Although these residual differences in the particles' velocities cause some small dispersion of the collective while streaming through the container [42], the dispersed collective will be collected again by a granular collapse, when it reaches the opposite container wall at a phase when the wall is accelerating inwards, that is, towards the approaching particles. For this regime the term collect-and-collide was coined [35]. A corresponding model description [36] explained the behavior of the granular damper including its two modes of operation and the corresponding dissipative properties in quantitative agreement with experiments. This model also explains why the amplitude of a spring with an attached granular damper decays nearly linearly in time [43], which was a long standing question. Under certain conditions, a granulate consisting of as few as 4 particles shows the collective behavior described above [37].

Under weak assumptions, the collect-and-collide and gas regimes are separated by a threshold amplitude of the oscillation:

$A_{0}=\frac{L_{g}}{\pi}$, 


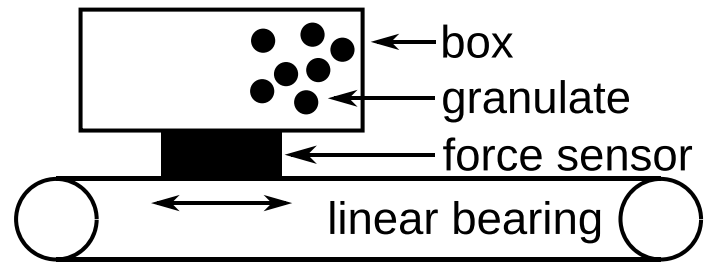

Fig. 1 Sketch of the experimental setup. For clarity, only one box is shown, for details see text

where $L_{g}$ is the free space inside the container which the cluster has to travel through between opposite walls of the container. Interestingly, the theory predicts that the value of $A_{0}$ is independent of the frequency of the oscillation and also material properties, in agreement with experimental results and numerical particle simulations [35-37]. For details of the derivation of Eq. (1) see [36]. Since the energy dissipation rate of both regimes is very different, the value of $A_{0}$ can be determined experimentally by measuring the power which is dissipated by the granular damper as a function of the vibration amplitude.

\section{Experimental setup and data processing}

\subsection{Experiment}

The experimental setup, sketched in Fig. 1, follows the same design as that employed in [36]: a cuboidal box constructed from $4 \mathrm{~mm}$-thick polycarbonate plates is filled with granular material and mounted on a linear bearing driven by a linear actuator. A force sensor is mounted between the wagon of the linear rail and the box, allowing measurement of the force $F(t)$ needed to impose sinusoidal motion

$x(t)=A \cos \left(\omega t+\varphi_{x}\right)$

on the box, where $A$ and $\omega$ are, respectively, the amplitude and the angular frequency of the motion.

A total of four such boxes are housed in the experimental rack. Each pair of boxes is mounted on a single linear rail and driven in anti-parallel orientation, such that the total momentum of each pair adds up to zero in order to minimise the vibrations exerted upon the experimental rack. Further, a Hall-effect based position encoder is mounted on each linear rail to measure the instantaneous position $x(t)$ of the box. Each box is monitored by a camera $(640 \times 480$ pixels at $240 \mathrm{fps}$ ) from the side (same perspective as the sketch in Fig. 1) and each pair of boxes is monitored by a high-speed camera $(1280 \times 1024$ pixels at $500 \mathrm{fps})$ from the top.

To exclude the sedimenting effect of gravity, this experiment has been performed in an aircraft performing parabolic
Table 1 Sample systems used in the experiments

\begin{tabular}{lllll}
\hline Sample no & Filling material & Mass $m(\mathrm{~g})$ & $\begin{array}{l}\text { Number of } \\
\text { particles } N\end{array}$ & $\begin{array}{l}\text { Empty } \\
\text { space } L_{g} \\
(\mathrm{~mm})\end{array}$ \\
\hline 1 & Glass & 24.4 & 260 & 94 \\
2 & Glass & 24.4 & 260 & 94 \\
3 & Glass & 24.4 & 260 & 94 \\
4 & Steel & 63.8 & 239 & 95 \\
5 & Steel & 126.3 & 473 & 89 \\
\hline
\end{tabular}

All boxes have the same inner dimensions of $100 \times 50 \times 50 \mathrm{~mm}^{3}$ and are filled with beads of $4 \mathrm{~mm}$ diameter. Samples 1-3 use glass beads, boxes 4 and 5 steel spheres. The measurements of boxes 1-3 were performed during the 31 st DLR Parabolic Flight Campaign and measurements of boxes 4 and 5 were performed during the 54th ESA Parabolic Flight Campaign [36]

flights, where in each parabola approximately $22 \mathrm{~s}$ of microgravity is achieved. Prior to each parabola, the apparatus is set to oscillate at desired amplitudes and a frequencies. At the same time, the recording of the force and the positional data is initiated at sample rate $10 \mathrm{kHz}$, and the cameras are triggered to record throughout the interval of microgravity. After the completion of each parabola, the oscillation is stopped and, if necessary, the boxes are swapped. This way we were able to study a variety of granular systems with different particle numbers and/or physical or geometrical properties. In order to obtain maximal information regarding the transition from the collect-and-collide to the gas regime, we focused on the range of amplitudes near the expected transition. The measurements presented below, were obtained with three distinct systems as described in Table 1, all containing the same filling volume, indicated by the empty space length, $L_{g}$.

Due to the box size and the amount of filling described by the empty space length, $L_{g}$, we expected the transition between the gas to the collective regime at amplitude $A_{0}=L_{g} / \pi \approx 29.9 \mathrm{~mm}$. Therefore, the range of amplitude in the experiment was chosen $20 \mathrm{~mm} \leq A \leq 40 \mathrm{~mm}$, to ensure the observation of the transition between the regimes.

\subsection{Data processing}

We obtain the dissipation rate of the granular dampers by averaging the time dependent power needed to set the damper in vibration, $P(t)$, over time. Note that the momentary value of $P(t)$ may be positive or negative, depending on the phase of the oscillation. We obtain

$$
\begin{aligned}
P(t) & =\frac{\mathrm{d} E(t)}{\mathrm{d} t}=F(t) \frac{\mathrm{d} x(t)}{\mathrm{d} t} \\
& =-F(t) A \omega \sin \left(\omega t+\varphi_{x}\right),
\end{aligned}
$$


where Eq. (2) was used. Expanding the force in harmonics with coefficients $F_{i}$ and $\varphi_{i}$, we write

$$
\begin{aligned}
P(t)= & -\sum_{n=1}^{\infty} F_{n} A \omega \cos \left(n \omega t+\varphi_{n}\right) \sin \left(\omega t+\varphi_{x}\right) \\
=\frac{1}{2} & \sum_{n=1}^{\infty} F_{n} A \omega\left[\sin \left((n-1) \omega t+\varphi_{n}-\varphi_{x}\right)\right. \\
& \left.\quad-\sin \left((n+1) \omega t+\varphi_{n}+\varphi_{x}\right)\right],
\end{aligned}
$$

where we exploit the identity $2 \cos (x) \sin (y)=\sin (x+y)-\sin (x-y)$. Averaging over one period of oscillation, we obtain the energy supplied to the system by the driving mechanism. Since we are in stationary state, this is identical to the energy dissipation rate,

$P_{\mathrm{diss}}=\frac{\omega}{2 \pi} \int_{t_{*}}^{t_{*}+\frac{2 \pi}{\omega}} P(t) \mathrm{d} t=\frac{A \omega}{2} F_{1} \sin \left(\varphi_{1}-\varphi_{x}\right)$,

where $t_{*}$ is an arbitrary time and we took into account that the integral vanishes for all terms in Eq. (4), except the $(n-1)$-term for $n=1$, corresponding to the frequency of the excitation.

Equation (5) thus allows us to determine the energy dissipation rate from the lowest order Fourier expansion of the measured driving force.

\section{Results}

To compute the energy dissipation rate of the granular damper, we measure the driving force as a function of time during the interval of weightlessness, compute the Fourier transform, and select the respective amplitude, $F_{1}$, and phase, $\varphi_{1}$, corresponding to the frequency $\omega$ of the driving. Then together with the amplitude and phase of the excitation, $A$ and $\varphi_{x}$, we determine $P_{\text {diss }}$ via Eq. (5).

Figure 2 shows the dissipated power of samples No. 1-3, described in Table 1 as functions of the vibration amplitude.

For amplitude $A \lesssim 30 \mathrm{~mm}$ the system is in the gas state where the energy dissipation rate is low, ( $P_{\text {diss }} \lesssim 7 \mathrm{~mW}$ ) while for $A \gtrsim 30 \mathrm{~mm}$ the system is in the collect-andcollide regime, characterized by larger dissipation rate, $P_{\text {diss }} \gtrsim 30 \mathrm{~mW}$. The transition amplitude agrees well with the prediction made by the model developed in [36], $A_{0} \approx 29.9 \mathrm{~mm}$, see Eq. (1). The transition between the regimes can also be seen from the images of the cameras. For sample 2, Fig. 3 illustrates the abrupt transition between the regimes through series of snapshots from one period of the vibration taken for amplitudes, $A=29.5 \mathrm{~mm}$ (left column) and $A=31.5 \mathrm{~mm}$, close to the transition point, $A_{0}$.

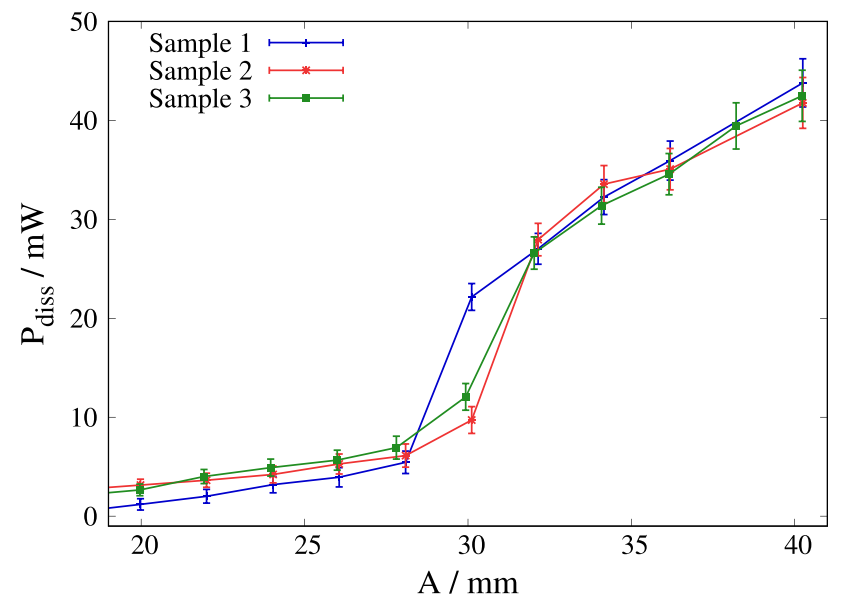

Fig. 2 Dissipated energy rate of the granular dampers No. 1-3, described in Table 1 as functions of the vibration amplitude

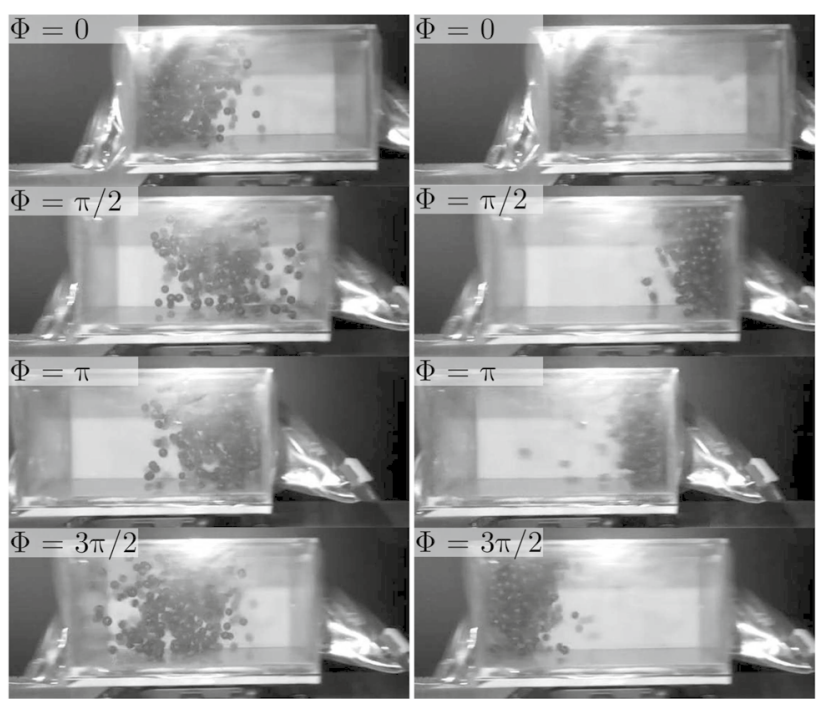

Fig. 3 Snapshots from the high speed video of sample 2 illustrating the sharp transition between the dynamical regimes of the granular damper, predicted at amplitude $A_{0}=29.9 \mathrm{~mm}$. The left column corresponds to $A=29.5 \mathrm{~mm}$, the right column to $A=31.5 \mathrm{~mm}$. $\Phi$ in the upper left indicates the phase of motion of the current snapshot. A high-speed recording showing a side by side comparison is provided in online supplemental material

For a more quantitative analysis of the transition between the dynamical regimes and the corresponding energy dissipation rates, we compare the results from the current experimental campaign presented in Fig. 3 with earlier data obtained during the 54th ESA parabolic flight where the energy dissipation rate of an equally sized granular damper was studied, but filled with varying masses of $4 \mathrm{~mm}$ steel beads (for details see [36]). For the comparison, we scaled the data in the same way as in the presentation of [37], namely the amplitude of the vibration was scaled by 


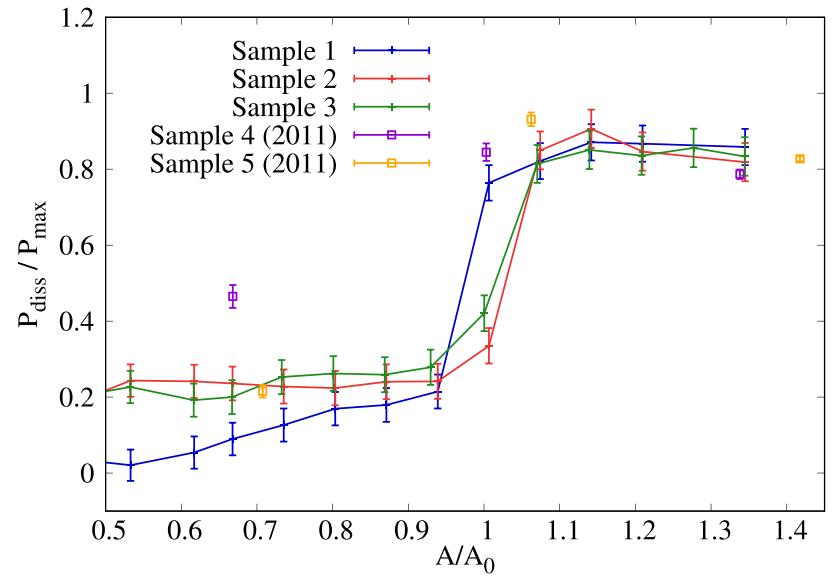

Fig. 4 Normalised energy dissipation rate as a function of the normalized amplitude of the vibration: Symbols connected by lines show current data while data represented only by symbols correspond to systems studied during the 54th ESA parabolic flight campaign in $2011[36]$

the amplitude $A_{0}=\frac{L_{g}}{\pi}$, such that the value $A / A_{0}$ discriminates the amplitudes corresponding to the gas state from the amplitudes corresponding to the collect-and-collide state. Second, the energy dissipation rate was scaled by the maximum energy dissipation rate which the system can possibly dissipate, $P_{\max }=\frac{2}{\pi} m A^{2} \omega^{3}$ similar to [36]. Figure 4 shows the current results superimposed by the results from the 54 th ESA parabolic flight, scaled in the described way.

We notice a very good quantitative agreement between the data from [36] and the current data. Deviations such as the point from sample No. 4 at $A / A_{0} \approx 0.67$ may be attributed to the coarse grained representation due to the sparse data in the range $A \approx A_{0}$ available in the earlier data set. While both sets of data obtained in independent experiments support one another, the new set clearly supports our conjecture made in [36], regarding the numerical value of the transition amplitude, $A_{0}$, and the fact that the transition is not smooth but occurs abruptly when $A$ approaches $A_{0}$.

\section{Summary}

When a granular damper is vibrated under conditions of microgravity, the energy dissipation rate depends strongly on the amplitude of the vibration, $A$, but shows no significant dependence on the frequency. When slowly increasing the amplitude, at the transition amplitude $A_{0}$, the granulate leaves the gas state characteristic for $A \lesssim A_{0}$ and assumes a different dynamical state called collect-and-collide regime characterized by collective motion of the particles. The transition between the dynamical states corresponds to an abrupt change of the energy dissipation rate. These statements were the main results of an experiment performed during the 54th ESA parabolic flight in 2011 and published in [36]. Since the theoretical model for $A_{0}$ was not available at this time, the amplitude was swept over a wide range of values such that the interesting range in the neighborhood of $A_{0}$ was covered only by a few data points, for details see [36]. Therefore, one could still have doubts about the statements above, thus, to refine the data in the range $A \approx A_{0}$ with the aim to characterize the transition between the regimes in more detail was the aim and motivation of the current study. The current experiments obtained during the 31st DLR parabolic flight campaign resolve the region around the transition amplitude, $A \approx A_{0}$ in much greater detail than previous measurements. As a result, the data support our conjecture made in [36], regarding the numerical value of the transition amplitude, $A_{0}$, and the fact that the transition is not smooth but occurs abruptly when $A$ approaches $A_{0}$.

Acknowledgements Open Access funding provided by Projekt DEAL. We gratefully thank the German Aerospace Center (DLR) and the European Space Agency (ESA) for funding the parabolic flight campaigns. We further gratefully acknowledge the support via DLR project Granada 50WM1752. The presented data were recorded during the 31 st DLR and the 54th ESA parabolic flight campaign in Bordeaux.

\section{Compliance with ethical standards}

Conflict of interest The authors declare that they have no conflict of interest.

Open Access This article is licensed under a Creative Commons Attribution 4.0 International License, which permits use, sharing, adaptation, distribution and reproduction in any medium or format, as long as you give appropriate credit to the original author(s) and the source, provide a link to the Creative Commons licence, and indicate if changes were made. The images or other third party material in this article are included in the article's Creative Commons licence, unless indicated otherwise in a credit line to the material. If material is not included in the article's Creative Commons licence and your intended use is not permitted by statutory regulation or exceeds the permitted use, you will need to obtain permission directly from the copyright holder. To view a copy of this licence, visit http://creativecommons.org/licenses/by/4.0/.

\section{References}

1. Pöschel. T., Schwager, G., Salueña, C.: Hand-held medical instrument. PCT Patent 2009/056126 (2009)

2. Heckel, M., Sack, A., Kollmer, J.E., Pöschel, T.: Granular dampers for the reduction of vibrations of an oscillatory saw. Phys. A 391, 4442-4447 (2012)

3. Ashley, S.: A new racket shakes up tennis. Mech. Eng. 117, 80-81 (1995)

4. Kielb, R., Macri, F.G., Oeth, D., Nashif, A.D., Macioce, P., Panossian, H., Lieghley, F.: Advanced damping systems for fan and compressor blisks. In: Proceedings of the 4th National Turbine Engine High Cycle Fatigue Conference, Monterey, CA (1999) 
5. Panossian, H.V.: Structural damping enhancement via nonobstructive particle damping technique. ASME J. Vibr. Acoust. 114, 101-105 (1992)

6. Xu, Z.W., Wang, M.Y., Chen, T.N.: An experimental study of particle damping for beams and plates. J. Vib. Acoust. 126, 141-148 (2004)

7. Norcross, J.C.: Dead-blow hammer head. U.S. Patent No. 3343576 (1967)

8. Salueña, C., Pöschel, T., Esipov, S.E.: Dissipative properties of vibrated granular materials. Phys. Rev. E 59, 4422-4425 (1999)

9. Salueña, C., Esipov, S.E., Pöschel, T., Simonian, S.: Dissipative properties of granular ensembles. SPIE 3327, 23 (1998)

10. Mao, K., Wang, M.Y., Xu, Z., Chen, T.: Dem simulation of particle damping. Powder Technol. 142, 154-165 (2004)

11. Mao, K., Wang, M.Y., Xu, Z., Chan, T.: Simulation and characterization of particle damping in transient vibrations. J. Vib. Acoust. 126, 202-211 (2004)

12. Chen, T., Mao, K., Huang, X., Wang, M.Y.: Dissipation mechanisms of nonobstructive particle damping using discrete element method. SPIE 4331, 294-301 (2001)

13. Bai, X.-M., Shah, B., Keer, L.M., Wang, Q.J., Snurr, R.Q.: Particle dynamics simulation of a piston-based particle damper. Powder Technol. 189, 115-125 (2009)

14. Bai, X.-M., Keer, L.M., Wang, Q.J., Snurr, R.Q.: Investigation of particle damping mechanism via particle dynamics simulation. Gran. Mat. 11, 417-429 (2009)

15. Falcon, É., Wunenburger, R., Évesque, P., Fauve, S., Chabot, C., Garrabos, Y., Beysens, D.: Cluster formation in a granular medium fluidized by vibrations in low gravity. Phys. Rev. Lett. 83, 440 (1999)

16. Évesque, P., Falcon, É., Wunenburger, R., Fauve, S., LecoutreChabot, C., Garrabos, Y., Beysens, D.: Gas-cluster transition of granular matter under vibration in microgravity. Micrograv. Res. Appl. Phys. Sci. Biotechnol. 454, 829 (2001)

17. Yoon, D.K., Arnarson, B.Ö., Jenkins, J.T.: Granular dissipative gas in weightlessness: the ultimate case ofvery low densities and how to measure accurately restitution coefficient and its dependence on speed. In: Powders and Grains 2005, Two Volume Set, pp. 1157-1162. CRC Press (2005)

18. Grasselli, Y., Bossis, G., Goutallier, G.: Velocity-dependent restitution coefficient and granular cooling in microgravity. Europhys. Lett. 86, 60007 (2009)

19. Zeng, X., Agui, J., Nakagawa, M.: Wave velocity measurement in granular materials under micro-gravity. In: 43rd AIAA Aerospace Sciences Meeting and Exhibit (2005)

20. Évesque, P., Palencia, F., Lecoutre-Chabot, C., Beysens, D., Garrabos, Y.: Granular gas in weightlessness: the limit case of very low densities of non interacting spheres. Micrograv.-Sci. Technol. 16, 280-284 (2005)

21. Harth, K., Kornek, U., Trittel, T., Strachauer, U., Höme, S., Will, K., Stannarius, R.: Granular gases of rod-shaped grains in microgravity. Phys. Rev. Lett. 110, 144102 (2013)

22. Yan-Pei, C., Évesque, P., Hou, M.: Breakdown of energy equipartition in vibro-fluidized granular media in micro-gravity. Chin. Phys. Lett. 29, 074501 (2012)

23. Grasselli, Y., Bossis, G., Morini, R.: Translational and rotational temperatures of a $2 \mathrm{~d}$ vibrated granular gas in microgravity. Eur. Phys. J. E 38, 8 (2015)

24. Feitosa, K., Menon, N.: Breakdown of energy equipartition in a $2 d$ binary vibrated granular gas. Phys. Rev. Lett. 88, 198301 (2002)

25. Windows-Yule, C.R.K., Parker, D.J.: Boltzmann statistics in a three-dimensional vibrofluidized granular bed: Idealizing the experimental system. Phys. Rev. E 87, 022211 (2013)

26. Falcon, É., Aumaître, S., Évesque, P., Palencia, F., LecoutreChabot, C., Fauve, S., Beysens, D., Garrabos, Y.: Collision statistics in a dilute granular gas fluidized by vibrations in low gravity. Europhys. Lett. 74, 830 (2006)
27. Leconte, M., Garrabos, Y., Falcon, É., Lecoutre-Chabot, C., Palencia, F., Évesque, P., Beysens, D.: Microgravity experiments on vibrated granular gases in a dilute regime: non-classical statistics. J. Stat. Mech: Theory Exp. 2006, P07012 (2006)

28. Hou, M., Liu, R., Zhai, G., Sun, Z., Lu, K., Garrabos, Y., Évesque, P.: Velocity distribution of vibration-driven granular gas in Knudsen regime in microgravity. Micrograv. Sci. Technol. 20, 73 (2008)

29. Tatsumi, S., Murayama, Y., Hayakawa, H., Sano, M.: Experimental study on the kinetics of granular gases under microgravity. J. Fluid Mech. 641, 521-539 (2009)

30. Opsomer, E., Ludewig, F., Vandewalle, N.: Phase transitions in vibrated granular systems in microgravity. Phys. Rev. E 84, 051306 (2011)

31. Harth, K., Trittel, T., May, K., Wegner, S., Stannarius, R.: Threedimensional (3d) experimental realization and observation of a granular gas in microgravity. Adv. Space Res. 55, 1901-1912 (2015)

32. Huang, Y., Zhu, C., Xiang, X., Mao, W.: Liquid-gas-like phase transition in sand flow under microgravity. Micrograv. Sci. Technol. 27, 155-170 (2015)

33. Noirhomme, M., Cazaubiel, A., Darras, A., Falcon, É., Fischer, D., Garrabos, Y., Lecoutre-Chabot, C., Merminod, S., Opsomer, E., Palencia, F., Schockmel, J., Stannarius, R., Vandewalle, N..: Threshold of gas-like to clustering transition in driven granular media in low-gravity environment. Europhys. Lett. 123, 14003 (2018)

34. Aumaître, S., Behringer, R.P., Cazaubiel, A., Clément, E., Crassous, J., Durian, D.J., Falcon, E., Fauve, S., Fischer, D., Garcimartín, A., Garrabos, Y., Hou, H., Jia, X., Lecoutre, C., Luding, S., Maza, D., Noirhomme, M., Opsomer, E., Palencia, F., Pöschel, T., Schockmel, J., Sperl, M., Stannarius, R., Vandewalle, N., Yu, P.: An instrument for studying granular media in low-gravity environment. Rev. Sci. Instrum. 89, 075103 (2018)

35. Bannerman, M.N., Kollmer, J.E., Sack, A., Heckel, M., Müller, P., Pöschel, T.: Movers and shakers: Granular damping in microgravity. Phys. Rev. E 84, 011301 (2011)

36. Sack, A., Heckel, M., Kollmer, J.E., Zimber, F., Pöschel, T.: Energy dissipation in driven granular matter in the absence of gravity. Phys. Rev. Lett. 111, 018001 (2013)

37. Sack, A., Heckel, M., Kollmer, J. E, Pöschel, T.: Probing the validity of an effective-one-particle description of granular dampers in microgravity. Granul. Matter 17, 73-82 (2015)

38. Heckel, M., Sack, A., Kollmer, J. E, Pöschel, T.: Fluidization of a horizontally driven granular monolayer. Phys. Rev. E 91, 062213 (2015)

39. Avila, K., Steub, L., Pöschel, T.: Liquidlike sloshing dynamics of monodisperse granulate. Phys. Rev. E 96, 040901 (2017)

40. McNamara, S., Young, W. R.: Inelastic collapse and clumping in a one-dimensional granular medium. Phys. Fluids A 4, 496-504 (1992)

41. Olafsen, J.S., Urbach, J. S.: Clustering, order, and collapse in a driven granular monolayer. Phys. Rev. Lett. 81, 4369 (1998)

42. Kollmer, J.E., Tupy, M., Heckel, M., Sack, A., Pöschel, T.: Absence of subharmonic response in vibrated granular systems under microgravity conditions. Phys. Rev. Appl. 3, 024007 (2015)

43. Kollmer, J.E., Sack, A., Heckel, M., Pöschel, T.: Relaxation of a spring with an attached granular damper. New J. Phys. 15, 093023 (2013)

Publisher's Note Springer Nature remains neutral with regard to jurisdictional claims in published maps and institutional affiliations. 